\title{
Effect of Rare Earth (Sm) ion Substitution on Structural, Dielectric, and Transport Properties of PZT Ceramics
}

\author{
Kanay Barik ${ }^{1}$, Rajiv Kumar ${ }^{2}$ \\ ${ }^{1}$ Research Scholar, Kolhan University, India \\ ${ }^{2}$ Jamshedpur Workers College, Jamshedpur 831012, India
}

\begin{abstract}
Solid solutions of $\mathrm{Pb}_{1-x} \mathrm{Sm}_{x}\left(\mathrm{Zr}_{0.4} \mathrm{Ti}_{0.6}\right)_{1-x / 4} \mathrm{O}_{3}(\mathrm{PSZT})(x=0.00,0.04,0.08$ and 0.12$)$ were synthesized by mixed oxide method at high temperature. The formation of single - phase compounds were confirmed by $X$-ray diffraction studies which were found to be in tetragonal phase at room temperature. The temperature dependence of dielectric response of the samples at selected frequencies has exhibit their phase transition well above the room temperature. The variation of ac conductivity with temperature reveals that their conduction process is of mixed type.
\end{abstract}

Keywords: PZT, Diffraction, Conductivity, Dielectric, Temperature

\section{Introduction}

There has been considerable interest in the solid solutions of lead zirconate-titanate, $\mathrm{Pb}\left(Z r_{1-y} T i_{y}\right) O_{3}$ (PZT) with a per perovskite $\mathrm{ABO} 3$ type $(\mathrm{A}=$ mono or divalent, $\mathrm{B}=$ trihexavalent ion) structure due to its possible forefront applications in the areas of research as well as in industrial applications [1-6]. A considerable amount of works have been done on modified PZT ceramics prepared from hightemperature solid-state reaction technique. It is found that the properties of PZT are very much sensitive to its compositional fluctuations near the morph tropical phase boundary (MPB), particle size, doping, calcinations and sintering temperature. Now days, with suitable modification in the compound, it is widely used for actuators, pyroelectric detectors, transducers, electro - optic, ferroelectric random access memory, sensors, etc. The physical properties and device parameters of PZT - based compounds are greatly influenced by chemical substitutions, synthesis process, and some other factors. It is well observed that the La-modified PZT has tremendous applications in electronics and electrooptics [6 - 8]. The literature survey on pure and modified PZT materials reveals that no systematic studies have been reported on physical properties and device parameters of Sm-substituted PZT (i.e., PSZT) with $\mathrm{Zr} / \mathrm{Ti}$ ratio 40/60 [9 13]. In view of the above, we have studied the effect of samarium substitution on structural, dielectric, and ac conductivity properties of PZT ( $\mathrm{Zr} / \mathrm{Ti}$ : 40/60) ceramics, which is reported here.

\section{Experimental Details}

The polycrystalline samples of $\mathrm{Sm}-$ modified PZT $P b_{1-x} \mathrm{Sm}_{x}\left(\mathrm{Zr}_{0.4} \mathrm{Ti}_{0.6}\right)_{1-x / 4} \mathrm{O}_{3}$ (where $x=0.00,0.04,0.08$, and 0.12 ) were prepared by a high- temperature solid-state reaction technique using high-purity $(99.9 \%)$ oxides (i.e., $\mathrm{PbO}, \mathrm{ZrO}_{2}, \mathrm{TiO}_{2}$, and $\mathrm{Sm}_{2} \mathrm{O}_{3}$ ) in a suitable stoichiometry with $3 \%$ more $\mathrm{PbO}$ (to compensate lead loss at high temperatures). The homogeneous mixed ingredients were calcined at an optimized temperature and time $\left(1100{ }^{\circ} \mathrm{C}, 10\right.$ hours) in an alumina crucible. The calcined powders, with small amount of polyvinyl alcohol (PVA) as binder, were converted into pellets at a pressure of $4 \times 10^{6} \mathrm{~N} / \mathrm{m}^{2}$ using hydraulic press. These pellets were sintered in an alumina crucible at an optimized temperature and time $\left(1200{ }^{\circ} \mathrm{C}, 10\right.$ hours) aiming to get nearly $97 \%$ of theoretical density. The $\mathrm{X}$-ray diffraction (XRD) data on the calcined powders were recorded using X-ray diffractometer (Rigaku Miniflex, Japan) with $\lambda=1.5405^{\circ} \mathrm{A}$ in a wide range of Bragg's angles $2 \theta\left(20^{\circ} \leq 2 \theta \leq 80^{\circ}\right)$ at a scanning rate of $3 \circ /$ minute. The dielectric data of the materials were obtained on silver electroded samples using phase sensitive multimeter (PSM; Model 1735) in a wide range of frequency $\left(10^{2}-10^{6} \mathrm{~Hz}\right)$ and temperature (room temperature $-500^{\circ} \mathrm{C}$ ) at a potential difference of $1 \mathrm{~V}$ with the stabilized temperature at an interval of $2.5^{\circ} \mathrm{C}$.

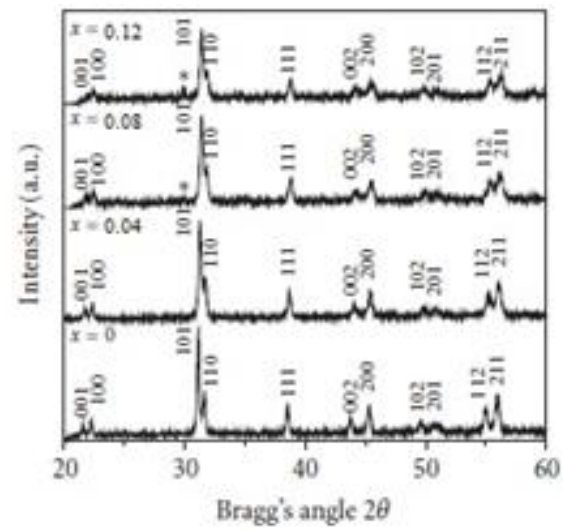

Figure 1: The comparison of XRD patterns of $P b_{1-x} S m_{x}\left(Z r_{0.4} T i_{0.6}\right)_{1-x / 4} O_{3}$ (for $x=0.00,0.04,0.08$, and 0.12 ).

\section{Results and Discussion}

\subsection{Structural Analysis}

The nature of room temperature XRD patterns of $\mathrm{Pb}_{1-x} \mathrm{Sm}_{x}\left(\mathrm{Zr}_{0.4} \mathrm{Ti}_{0.6}\right)_{1-x / 4} \mathrm{O}_{3}$ (PSZT) with $x=0.00,0.04$, 


\section{International Journal of Science and Research (IJSR) \\ ISSN (Online): 2319-7064}

Index Copernicus Value (2013): 6.14 | Impact Factor (2014): 5.611

0.08 , and 0.12 (Figure 1) as compared to the reported ones confirms the formation of single phase with tetragonal crystal structure $[14,15]$. All the reflection peaks were indexed in tetragonal crystal system using computer software POWDMULT [16]. On the basis of best agreement between the observed (obs) and the calculated (cal) $d$ spacing (i.e., $\sum \Delta d=d_{\mathrm{obs}}-d_{\mathrm{cal}}=$ minimum), all the PSZT compounds were found to be in tetragonal crystal system with their refined lattice parameters given in Table 1 . In the XRD patterns, there is an additional peak (for $x \geq 0.08$ ) usually referred as secondary or pyrochlore phase $[17,18]$. Though these peaks are undesirable, it is some time essential for formation of the perovskites [19]. The percentage of pyrochlore phase in PSZT for $x=0.08$ and 0.12 was estimated as $3 \%$ and $7 \%$, respectively [20].

\subsection{Dielectric Study}

The variation of relative dielectric constant $\left(\varepsilon_{r}\right)$ of PSZT (having Sm contents $x=0.00,0.04,0.08$, and 0.12) with temperature at selected frequencies $\left(10^{3}-10^{6} \mathrm{~Hz}\right)$ is shown in Figure 2. It is found that $\varepsilon_{r}$ decreases on increasing frequency which indicates a normal behavior of the ferroelectric and/or dielectric materials. The higher values of $\varepsilon_{r}$ at lower frequency are due to the simultaneous presence of all types of polarizations (space charge, dipolar, ionic, electronic, etc.) which is found to decrease with the increase in frequency. At high frequencies $\left(>10^{12} \mathrm{~Hz}\right)$ electronic polarization only exists in the materials. When temperature of PSZT samples is increased, er first increases slowly and then rapidly up to a maximum value (cmax). Temperature of the material corresponding to $\varepsilon_{\max }$ is called Curie or critical temperature $(\mathrm{Tc})$. As at this $\mathrm{Tc}$, phase transition takes place between ferroelectric-pyroelectric phases so it is also called transition temperature. At the higher temperature $(\geq \mathrm{Tc})$, the space charge polarization originates due to mobility of ions and imperfections in materials and thus contributes to a sharp increase in $\varepsilon_{\mathrm{r}}[21,22]$. The value of $\varepsilon_{\max }$ is found to be highest for PZT. As Sm content in PSZT increases, the value of emax exhibits a sharp decrease for $\mathrm{x}=0.04$, then an increase for $x=0.08$, and again decrease for $x=0.12$. The value of Tc is found to be highest for PZT which decreases gradually on increasing Sm content in PSZT. However, for each PSZT samples Tc is found to be unaffected with the change in frequency supporting the nonrelaxor behavior of Sm-modified PZT. The values of $\varepsilon_{\max }$ and Tc of PSZT are compared in Table 1.

The frequency-temperature dependence of tangent loss (tan $\delta$ ) of PSZT is shown in Figure 2. With the increase in temperature, $\tan \delta$ is found to be very low and almost remains constant up to $T c$ beyond which it indicates a significant increase. The nature of variation of $\tan \delta$ at higher frequency and temperature can be explained by space-charge polarization. This $\tan \delta$ decreases with the increase in frequency as expected $[23,24]$.

\subsection{Ac Conductivity}

The ac conductivity $\left(\zeta_{\mathrm{ac}}\right)$ of PSZT for $x=0.00,0.04,0.08$, and 0.12 at frequency $10 \mathrm{kHz}$ was calculated using dielectric relation

$$
\zeta_{\mathrm{ac}}=\omega \varepsilon_{o} \varepsilon_{r} \tan \delta
$$

where $\omega$ is the angular frequency and $\varepsilon_{o}$ the permittivity of free space. Figure 3 shows an increasing trend of ac conductivity around Tc. A sharp maximum in $\zeta_{\mathrm{ac}}$ at $T c$ (observed by dielectric analysis) indicates a marked dispersion which may be due to the increase in polarizability. Above $T c$, the conductivity data appears to fall on a straight line exhibiting a typical behavior of the dc component of the conductivity [23]. The linear variation of $\zeta_{\text {ac }}$ over a wide range of temperature supports the existence of thermally activated transport properties in the materials following the Arrhenius equation:

$$
\zeta_{\mathrm{ac}}=\zeta_{0} \exp \left(-\frac{E_{a}}{K_{B} T}\right)
$$

where $\zeta_{o}$ is the pre-exponential factor, $K B$ the Boltzmann constant and $E a$ the activation energy. The value of activation energy ( $E a)$ of PSZT was found to be $0.93,0.57$, 1.45 , and 0.79 for $x=0.00,0.04,0.08$, and 0.12 , respectively, in the high-temperature pyroelectric phase which suggests its dependence on ionization level of oxygen vacancy [25].

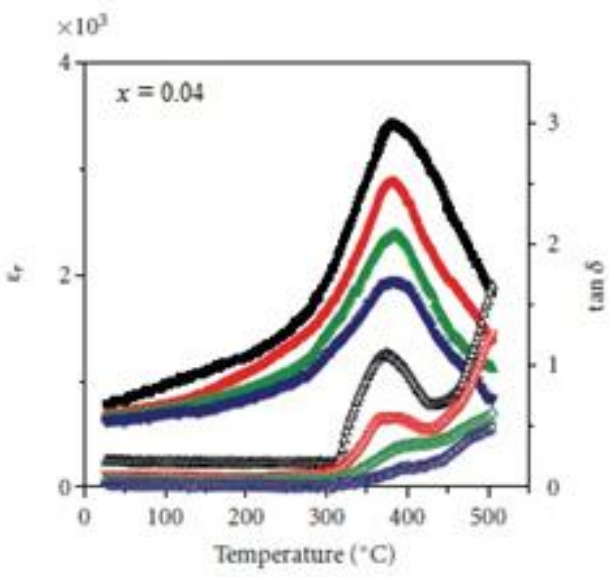

(b) 
International Journal of Science and Research (IJSR)

ISSN (Online): 2319-7064

Index Copernicus Value (2013): 6.14 | Impact Factor (2014): 5.611

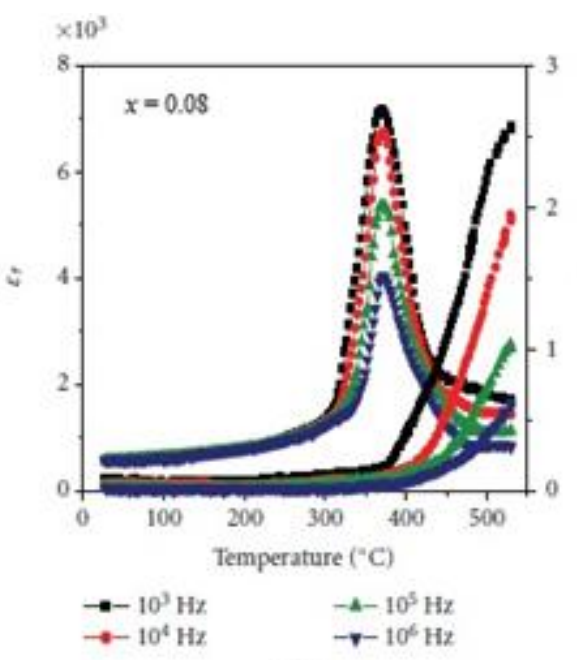

(c)

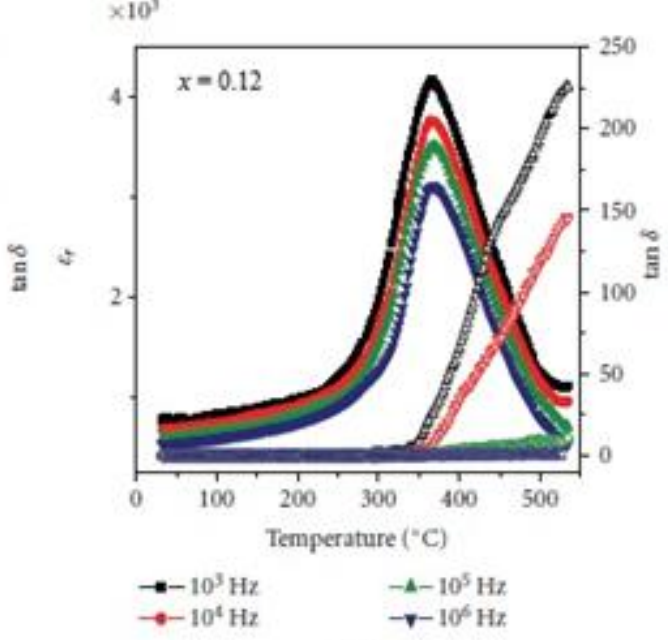

(d)

Figure 2: Temperature-frequency dependence of relative dielectric constant $\left(\varepsilon_{r}\right)$ and tangent loss $(\tan \delta)$ $P b_{1-x} S m_{x}\left(Z r_{0.4} T i_{0.6}\right)_{1-x / 4} O_{3}$ for $x=0.00,0.04,0.08$, and 0.12 .

Table 1: Comparison of the lattice parameters, $\varepsilon_{\max }$ and $T c$ of $\mathrm{Pb}_{1-x} \mathrm{Sm}_{x}\left(\mathrm{Zr}_{0.4} \mathrm{Ti}_{0.6}\right)_{1-x / 4} \mathrm{O}_{3}$ for $\mathrm{x}=0.00,0.04,0.08$ and 0.12

\begin{tabular}{|c|c|c|c|c|}
\hline & & Sm composition & & \\
\hline Parameters & $\mathrm{x}=0.00$ & $\mathrm{x}=0.04$ & $\mathrm{x}=0.08$ & $\mathrm{x}=0.12$ \\
\hline $\mathrm{a}$ & $4.0042(21)$ & $3.9966(50)$ & $3.9725(50)$ & $3.9785(50)$ \\
\hline $\mathbf{c}$ & $4.1348(21)$ & $4.1146(50)$ & $4.0844(50)$ & $4.1081(50)$ \\
\hline $\mathrm{c} / \mathrm{a}$ & $1.0326(21)$ & $1.02952(50)$ & $1.02816(50)$ & $1.0325(50)$ \\
\hline$\varepsilon_{\max }$ & 10850 & 3421 & 7186 & 4164 \\
\hline $\mathrm{T}(\mathrm{C})$ & $404 \pm 0.25$ & $383 \pm 0.25$ & $371 \pm 0.25$ & $364 \pm 0.25$ \\
\hline
\end{tabular}

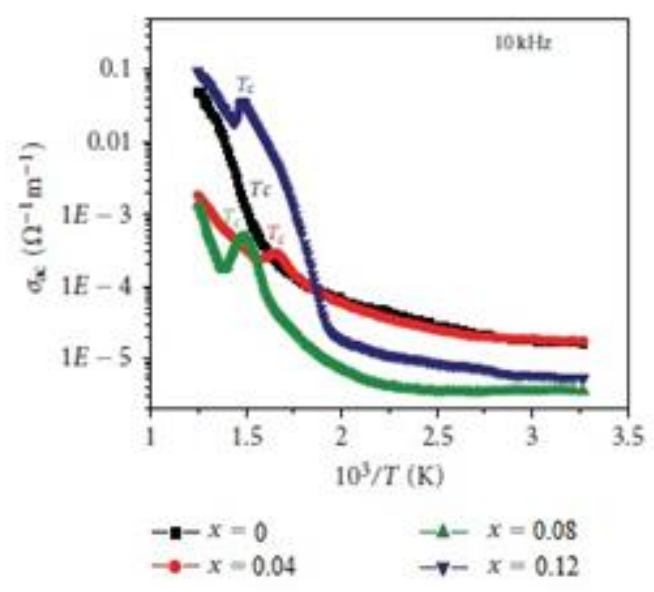

Figure 3: Temperature-Frequency dependence of ac conductivity $P b_{1-x} S m_{x}\left(Z r_{0.4} T i_{0.6}\right)_{1-x / 4} O_{3}$ for $\mathrm{x}=0.00$, $0.04,0.08$ and 0.12

\section{Conclusions}

Preliminary structural analysis using room temperature X ray diffraction data obtained from the calcined powders of polycrystalline samples of Sm-modified PZT (i.e., $\left.P b_{1-x} S m_{x}\left(Z r_{0.4} T i_{0.6}\right)_{1-x / 4} O_{3}\right)$ has confirmed their tetragonal phase with the presence of a small amount of pyrochlore phase during higher concentration of $\mathrm{Sm}(3 \%$ for $x=0.08$ and $7 \%$ for 0.12 ). Detailed study of dielectric properties of PSZT as a function of temperature at selected frequencies has exhibited that maximum or peak dielectric constant, tangent loss, and transition temperature are strongly dependent on Sm content in PSZT. The electrical conductivity (ac) of PSZT may not only due to singly ionized in low temperature (ferroelectric phase) region but also due to doubly ionized in the high-temperature region.

\section{References}

[1] Lines, M. E. and Glass, A. M., Principles and Applications of Ferroelectrics and Related Materials. Oxford University Press Oxford, 1977.

[2] Buchanan, R. C., Ceramic Materials for Electronics. Marcel Decker, New York, 1986.

[3] Smyth, A. P., Dielectric Behavior and Structure. McGraw-Hill Publishers, New York, 1955.

[4] Herbert, J. M., Ceramic Dielectrics and Capacitors. Gordon and Breach Science Publishers, New York, 1985.

[5] Cross, L. E., Ferroelectric materials for electromechanical transducer applications. Jpn. J. Appl. Phys., 1995, 34, 2525-2532.

[6] S. A. Mabud, "The morph tropic phase boundary in PZT solid solutions," Journal of Applied Crystallography, vol. 13, part 3, pp. 211-216, 1980.

[7] G. H. Haertling, "Piezoelectric and electro optic ceramics," in Ceramic Materials for Electronics, R. C. Buchanan, Ed., chapter 3, pp. 139-225, Marcel Dekker, New York, NY, USA, 1991.

[8] B.-S. Chiou, J. N. Kuo, and H. T. Dai, "The preparation of PLZT ceramics from a sol-gel process," Journal of Electronic Materials, vol. 19, no. 4, pp. 393-397, 1990.

[9] U. Chon, K.-B. Kim, H. M. Jang, and G.-C. Yi, "Fatigue-free samarium-modified bismuth titanate (Bi4- $x$ Sm $x$ Ti3O12) film capacitors having large spontaneous polarizations," Applied Physics Letters, vol. 79, no. 19, pp. 3137-3139, 2001.

[10]C. Pramila, T. C. Goel, and P. K. C. Pillai, "Investigations on the piezoelectric and structural properties of samarium doped lead zirconate titanate ceramics," Materials Science and Engineering B, vol. 26, no. 1, pp. 25-28, 1994.

[11] S. K. Pandey, O. P. Thakur, D. K. Bhattacharya, C. Prakash, and R. Chatterjee, "Structural and electrical properties of Sm3 + substituted PZT ceramics," Journal

\section{Volume 4 Issue 12, December 2015}




\section{International Journal of Science and Research (IJSR) \\ ISSN (Online): 2319-7064}

Index Copernicus Value (2013): 6.14 | Impact Factor (2014): 5.611

of Alloys and Compounds, vol. 468, no. 1-2, pp. 356$359,2009$.

[12] S. R. Shannigrahi and R. N. P. Choudhary, "Structural and electrical properties of sol-gel prepared $\mathrm{Sm}$ modified $\mathrm{Pb}(\mathrm{Zr} 0.60 \mathrm{Ti0}$.40)O3 ceramics," British Ceramic Transactions, vol. 101, no. 1, pp. 25-29, 2002.

[13] C. Prakash and J. K. Juneja, "Investigations on Sm- and Nb substituted PZT ceramics," Modern Physics Letters $B$, vol. 20, no. 29, pp. 1879-1882, 2006.

[14] A. K. Tripathi, T. C. Goel, and C. Prakash, "Preparation of 4:55:45 samarium doped PZT films by sol-gel technique and their characterization," Materials Science and Engineering B, vol. 96, no. 1, pp. 19-23, 2002.

[15] R. Khazanchi, S. Sharma, and T. C. Goel, "Effect of rare earth Europium substitution on themicrostructure, dielectric, ferroelectric and pyroelectric properties of PZT ceramics," Journal of Electro ceramics, vol. 14, no. 2, pp. 113-118, 2005.

[16]E. Wu, "POWDMULT: an interactive powder diffraction data interpretation and indexing programmed version 2.1," School of Physical Sciences, Flinders' University of South Australia Bradford Park, SA, and Australia.

[17] S. R. Shannigrahi, R. N. P. Choudhary, and H. N. Acharya, "X-ray, SEM and dielectric studies of Gdmodified sol-gel prepared lead zirconate-lead titanate solid solution," Materials Letters, vol. 39, no. 6, pp. 318-323, 1999.

[18] J. Lian, L.Wang, J. Chen, et al., "The order-disorder transition in ion-irradiated pyrochlore," Acta Materialia, vol. 51, no. 5, pp. 1493-1502, 2003.

[19] A. H. Carim, B. A. Tuttle, D. H. Doughty, and S. L. Martinez, "Microstructure of solution-processed lead zirconate titanate (PZT) thin films," Journal of the American Ceramic Society, vol. 74, no. 6, pp. 14551458, 1991.

[20] S. L. Swartz and T. R. Shrout, "Fabrication of perovskite lead magnesium niobate," Materials Research Bulletin, vol. 17, no. 10, pp. 1245-1250, 1982.

[21]J. C. Anderson, in Dielectrics, Chapman \& Hall, London, UK, 1964.

[22]C. J. F. Bottchar, Theory of Electric Polarization, Elsevier, Amsterdam, The Netherlands, 1952.

[23]M. E. Lines and A. M. Glass, Principles and Applications of Ferroelectrics and Related Materials, Oxford University Press, London, UK, 1977.

[24] S. R. Shannigrahi, F. E. H. Tay, K. Yao, and R. N. P. Choudhary, "Effect of rare earth ( $\mathrm{La}, \mathrm{Nd}, \mathrm{Sm}, \mathrm{Eu}, \mathrm{Gd}$, $\mathrm{Dy}, \mathrm{Er}$ and $\mathrm{Yb}$ ) ion substitutions on the micro structural and electrical properties of sol-gel grown PZT ceramics," Journal of the European Ceramic Society, vol. 24, no. 1, pp. 163-170, 2004.

[25]C. Ang, Z. Yu, and L. E. Cross, "Oxygen-vacancyrelated low frequency dielectric relaxation and electrical conduction in Bi: SrTiO3," Physical Review B, vol. 62, no. 1, pp. 228-236, 2000. 